\title{
Mental health of colombinn general proctitioners during the pandemic: reflections from a brief survey
}

\author{
Mayra Alejandra Martinez Miranda* \\ Jorge Homero Wilches Visbal**
}

\begin{abstract}
Citar este artículo así:
Martínez MA, Wilches $\mathrm{JH}$. Mental health of colombian general practitioners during the pandemic: reflections from a brief survey. Hacia. Promoc. Salud. 2021; 26(1): 17-19 DOI: 10.17151/ hpsal.2021.26.1.3
\end{abstract}

\section{Dear Editor,}

Since before the COVID-19 pandemic, the fact that physicians and other health professionals are subjected to different states of emotional and physical fatigue associated with their work has been known (1). Recent studies have found a significant incidence of depression in this population compared to others, but this reality has been insufficiently addressed by state agencies (2). In the context of a pandemic, a greater emotional impact on health workers is predictable due to the stress of this unprecedented situation. Once this thesis is verified, the need to address the underlying risks that health personnel have regarding the development of various mental health problems associated with anxiety, depression or suicide is raised by means of efficient preventive measures that stipulate protocols aimed at improving the work environment (2).

Indeed, some countries where public health policies have been implemented to protect the mental health of physicians and other health professionals show considerable decrease in cases of intolerance by the population towards the health personnel as well as a general increase in confidence in medical judgment (3). In the practice of medicine during the pandemic, various populations of physicians have had in common stressors caused by workload increase, low work experience, collapse of health-providing institutions due to a large number of critical patients, and being part of the front-line physicians, all this in line with higher scores on the Depression Anxiety Stress Scale (DASS-21) observed in previous studies (4). These factors worsen in young people and women because they are a higher incidence of depressive and anxiety disorders in these populations (5). General practitioners in Colombia have been affected by a series of unusual situations in their professional practice during the COVID-19 pandemic. Some of those many reported incidents have been related to physical and verbal attacks, untimely payments

\footnotetext{
* General Physician. E.P.S. Sanitas, Medellín, Colombia. Email: mayamartinez@epssanitas.com

(D) orcid.org/0000-0003-2373-0711.

**Physical Engineer, Doctor in Physics Applied to Medicine and Biology. Occasional Full Time Professor, Faculty of Health Sciences, Universidad del Magdalena, Santa Marta, Colombia. Email: jhwilchev@gmail.com.

(D) orcid.org/0000-0003-3649-5079.
} 
and deficiencies in the provision of personal protection elements by employers, increased workload and witnessing massive deaths of COVID-19 patients. These events represent an emotional burden that can affect the performance of the medical practice and generate a deterioration in the quality of life $(6,7)$. To explore the stressful situations to which Colombian physicians have been subjected in the exercise of their work during the pandemic, a psychological survey was designed and sent to about 600 Colombian physicians. The survey questions were associated with the degree of satisfaction of physicians with respect to their employers and the personal protection elements provided by them, as well as with the presence of mood disorders and physical aggression.

The survey was completed only by 35 out of the 600 physicians to whom it was sent, although they had a 2-month margin (July-August 2020) to answer it. The results are listed in the table below.

Table. Percentage of surveyed physicians who responded positively to questions related to various stressful situations during the COVID-19 pandemic.

\begin{tabular}{lc}
\hline \multicolumn{1}{c}{ Stressful situations } & Percentage of Positive Responses (\%) \\
\hline Symptoms associated with mood disorders & 97.0 \\
\hline Suicidal ideas & 11.4 \\
\hline Dissatisfaction with the employer & 77.8 \\
\hline Deficiency in personal protection elements. & 66.7 \\
\hline Victims of physical assault & 54.3 \\
\hline
\end{tabular}

According to the results, $54.3 \%$ of the surveyed physicians said they had been assaults victims, $77.8 \%$ were not satisfied with their working conditions, $66.7 \%$ indicated that they did not receive the adequate personal protection elements and this generated fear of contagion. Symptoms associated with mood disorders were reported by $97 \%$ of those surveyed, $11.4 \%$ also stated that they had suffered from suicidal ideation at some point during the pandemic. More than $50 \%$ of the participants experienced some type of altered mental state or physical violence derived from situations related to the pandemic. Furthermore, almost $100 \%$ of those who completed the survey stated that they had suffered symptoms associated with mood disorders.

The emotional burden of health professionals in Colombia involves unusual situations of psychological and physical stress derived from the problems in the types of hiring, since this aspect usually lacks inspection and control by the governmental entities. Additionally, the growing events of physical and verbal violence as a result of the intolerance of certain social sectors, the discredit or unfounded hoaxes about medical criteria and the scant security surveillance of some clinics and hospitals have also caused distress. The increasing workload due to the emergency triggered by the pandemic is another explanatory factor for the recurring changes in the moods of health professionals. One of the lessons that COVID-19 has taught is the imperative need to generate effective policies that protect physicians, especially those who begin their professional career, both in its physical and mental dimensions. 


\section{Bibliographical references}

1. Pereda-Torales L, Celedonio FGM, Vásquez MTH, Zamora MIY. Síndrome de burnout en médicos y personal paramédico. Salud Ment. 2009;32(5):399-404.

2. Alvarado-Socarras JL, Manrique-Hernández EF. Suicidio de médicos. Una realidad ignorada. Salud UIS. 18 de junio de 2019;51(3):194-6.https://doi.org/10.18273/revsal.v51n3-2019001

3. Wang C, Pan R, Wan X, Tan Y, Xu L, McIntyre RS, et al. A longitudinal study on the mental health of general population during the COVID-19 epidemic in China. Brain Behav Immun. July 2020;87:40-8.

4. Elbay RY, Kurtulmuş A, Arpacioğlu S, Karadere E. Depression, anxiety, stress levels of physicians and associated factors in Covid-19 pandemics. Psychiatry Res. August 2020;290:113130

5. Rodríguez-Rey R, Garrido-Hernansaiz H, Collado S. Psychological Impact of COVID-19 in Spain: Early Data Report. Psychol Trauma Theory, Res Pract Policy. 2020; 12(5), 550-552. http://dx.doi. org/10.1037/tra0000943

6. Bueno Ferrán M, Barrientos-Trigo S. Caring for the caregiver: The emotional impact of the coronavirus epidemic on nurses and other health professionals. Enferm Clin. May 2020. http:// dx.doi.org/10.1016/j.enfcli.2020.05.006

7. Erquicia J, Valls L, Barja A, Gil S, Miquel J, Leal-Blanquet J, et al. Impacto emocional de la pandemia del Covid-19 en los trabajadores sanitarios de uno de los focos de contagio más importantes de Europa. Med Clin (Barc). July 2020. https://dx.doi.org/10.1016\%2Fj.medcli.2020.07.006 